\title{
EFECTO DEL MÉTODO DE ESTIRAMIENTO P.N.F. Y ESTÁTICO EN LA MEJORA DE LA FLEXIBILIDAD DE LA ARTICULACIÓN DE LA CADERA EN PRACTICANTES DE KUNG FU
}

\author{
Juan Diego Zamora Salas y Walter Salazar Rojas \\ Escuela de Educación Física y Deportes \\ Universidad de Costa Rica, San José, Costa Rica \\ E-mail: juandiegozamora@hotmail.com
}

\begin{abstract}
Resumen
Zamora, J. D., y Salazar, W. (2001). Efecto del método de estiramiento P.N.F. y estático en la mejora de la flexibilidad de la articulación de la cadera en practicantes de Kung Fu. Revista en Ciencias del Ejercicio y la Salud, 1(2), 43-50. El presente estudio analizó el efecto de dos métodos de estiramiento (estático y P.N.F.) para mejorar el rango de movimiento en la articulación de la cadera en practicantes de Kung Fu Wing Chun, utilizando una prueba de flexibilidad de cadera. Se formaron tres grupos cada uno conformado por $n=10$, cuyas edades estaban comprendidas entre los 15 y 28 años. Los sujetos del Grupo Control les correspondía continuar con sus entrenamientos normalmente; y a los sujetos del Grupo B y Grupo C, les correspondía realizar la prueba de flexibilidad de la cadera utilizando el método estático y el método P.N.F. respectivamente. A los valores recolectados durante la prueba se les aplicó una Anova 3x3, Análisis de Efecto Simple para Grupos y Mediciones, además de un análisis de Tukey, en donde se encontró una mejora de los resultados tanto en el grupo que utilizó el método estático como para el que utilizó el método P.N.F., en comparación al grupo control, pero no existiendo una diferencia significativa entre ambos métodos, sólo entre el pre-test y post-test de ambos tratamientos. También se encontró una mayor retención de la mejora en el R.M.A.; en los sujetos que utilizaron el método P.N.F., cuatro semanas después de haberse suspendido éste método en comparación a los sujetos que utilizaron el método estático. PALABRAS CLAVE: Flexibilidad, Elasticidad, Método Estático, Método P.N.F., Cadera, Kung Fu, Artes Marciales
\end{abstract}

\section{INTRODUCCION}

En la actualidad la flexibilidad es aceptada como un importante componente del estado físico general, que permite reducir en un gran porcentaje el sufrir algún tipo de lesión durante la ejecución de algún movimiento, $\mathrm{y}$ como un medio para mejorar el rendimiento durante alguna actividad física o deportiva.

Para James y Hart (1990), la flexibilidad tiene un fuerte efecto no sólo en el movimiento sino también en la postura.

La mayoría de actividades físicas que hacemos diariamente requieren de cantidades normales de flexibilidad; sin embargo para la realización de deportes como la gimnasia, ballet, clavados, artes marciales y yoga se requiere de una mayor flexibilidad para lograr mejores desempeños.(Prentice y Bucher; 1988).

Actualmente la flexibilidad es definida como la máxima disponibilidad que tiene una articulación en su rango de movimiento.(Magnusson, Simonsen, Aagaard, Boesen, Johannsen, Kjaer; 1997). Hardy (1985), menciona que el aumento en el rango de movimiento articular es posible al incrementar la elasticidad en los músculos agonistas o disminuyendo la resistencia en los músculos antagonistas.

Para James y Hart (1990), la amplitud en el rango de movimiento articular es altamente hereditaria, aunque se puede aumentar hasta cierto grado especialmente antes de la adolescencia.

De acuerdo a James y Hart (1990), la flexibilidad de un individuo está determinada por los efectos combinados de dos características principales: (1)- Movilidad de la Articulación, que determina la máxima amplitud de movimientos alrededor de la articulación, la cual consta de la estructura ósea de la misma y de la longitud de los ligamentos conectivos que 
sostiene la articulación; y (2)- Extensión del Músculo, que está determinada por la longitud de los músculos (y sus tendones) que cruzan la articulación.

De acuerdo a Prentice y Bucher (1988), una persona con un rango de movimiento articular limitado probablemente disminuirá sus capacidades para poder llevar a cabo algún trabajo físico, ejercicio o hasta una simple tarea doméstica; además de que podría presentar una postura deficiente lo que especialmente en personas de la tercera edad resulta en una limitación para su libertad de movimiento.

También Prentice y Bucher (1988), consideran que la falta de flexibilidad puede contribuir o tal vez puede causar la mayoría de lesiones o condiciones crónicas, resultando muchas veces en movimientos extraños y descoordinados que probablemente predispongan a una persona a sufrir alguna lesión muscular.

Según James y Hart (1990), la flexibilidad es un importante factor en la prevención de lesiones tanto en la que tienen como origen torceduras y distenciones, o en aquellas a largo plazo que se hacen crónicas a causa de movimientos repetidos con mucha tensión. Magnusson y colegas (1997), afirman que las lesiones son producidas por una pasiva propiedad del músculo y una limitada flexibilidad. También proponen que para evitar las lesiones es importante realizar una rutina de ejercicios de estiramiento, los cuales permitan aumentar el rango de movimiento articular.

Los ejercicios de estiramiento pueden llevarse a cabo solos o combinados con otros, como parte del calentamiento del deportista, el cual debe estar diseñado para aumentar la elasticidad de los músculos.(Smith, 1994); aunque para Purcell y Hergenroeder (1994), los ejercicios de estiramiento no ayudan a prevenir lesiones en personas que no hayan sufrido lesiones previas.

De acuerdo a Magnusson y colegas (1997), la tolerancia al estiramiento que posea una persona, permite mejorar el rango de movimiento articular en comparación con de un estado pasivo del músculo; y que la propiedad del material del músculo esquelético durante el estiramiento está relacionado al aumento del colágeno.
La elasticidad de un músculo puede ser dividida en una fase dinámica en la cual la unidad tendón-músculo está pasivamente estirada, seguido por una fase estática en la cual un estiramiento constante del músculo-tendón es mantenida por algún tiempo.(Magnusson, y colegas; 1997).

James y Hart (1990), mencionan que las mujeres tienen más elasticidad que los hombres y los niños más que las mujeres; lo que afecta su grado de movilidad articular.

La flexibilidad se puede ir perdiendo con la edad ya que los tejidos conectivos, los tendones y los ligamentos se engruesan; sin embargo, es muy dificil determinar la extensión real de los efectos de la edad, debido a los cambios en los tipos y niveles de actividad que pasan las personas durante su vida. (James y Hart, 1990)

Entre los métodos más utilizados para incrementar el rango de movimiento articular tenemos el balístico (de acuerdo a Swinnen y Van Assche; 1988, también conocido como dinámico activo), el estático y las variaciones de la facilitación neuromuscular propioceptiva (cuyas siglas en inglés corresponden a P.N.F.).

El estiramiento balístico consiste en ejecutar movimientos rápidos con una parte del cuerpo que conforma un segmento de una articulación, los cuales producen tensión en un músculo o grupo muscular en particular y en el tejido conectivo que envuelve el músculo y articulación; el estiramiento estático consiste en producir una tensión constante en una

determinada articulación.(Etnyre y Lee; 1988). La P.N.F. es definida como una técnica de rehabilitación cuya finalidad es permitir y facilitar el desencadenamiento de los mecanismos neuromusculares, los cuales son estimulados por los propioceptores; por lo cual éste método es útil para el tratamiento de lesiones, recuperación postraumática y múltiples facetas del entrenamiento.(López, 1991).

En un estudio realizado por Lucas y Koslow (1984), se compararon los métodos de estiramiento más utilizados (balístico, estático y P.N.F.), para mejorar la flexibilidad en la articulación de la rodilla, al ser aplicados tales métodos en los músculos gastronemios, se comprobó una mejora significativa en la 
flexibilidad de tal articulación al ser utilizados los tres métodos de estiramiento. En 1985 Williford y Smith realizaron un estudio comparativo entre el método de estiramiento estático y el P.N.F., con el fin de mejorar la flexibilidad en la articulación de la cadera, sin encontrar diferencias significativas entre ambos métodos; sin embargo el estudio mostró diferencias significativas entre los resultados obtenidos en el pre-test y post-test de cada uno de los métodos utilizados. En su estudio Williford y col. (1985), concluyen que ambos métodos de estiramiento son igualmente de efectivos para incrementar la flexibilidad en la articulación de la cadera y que ninguno demostró ser más efectivo que el otro.

En un estudio realizado por Condon y Hutton (1987), no se encontró diferencia significativa entre el método de estiramiento estático y el P.N.F.; luego de ser aplicados en la articulación de la cadera tanto en hombres como en mujeres. Etnyre y Lee (1988), citan que en una gran mayoría de estudios se observan mejorías en la articulación de la cadera al utilizar el método P.N.F. en comparación con el método estático.

Para Swinnen y Van Assche (1988), el método más efectivo para aumentar el rango de movimiento articular es el P.N.F., en comparación a otros métodos conocidos.

Etnyre y Lee (1988), mencionan que estudios realizados en hombres utilizando el método de estiramiento estático y el método P.N.F., aplicados en la articulación de la cadera se encontraron diferencias significativas entre ambos métodos, en donde los mejores resultados se apreciaron en los sujetos que utilizaron el método P.N.F. como tratamiento, en comparación con los que utilizaron el método estático. También mencionan que cuando se aplicó uno de éstos métodos a grupos de sujetos en una sola sesión, se comprobó que al utilizar el método P.N.F. se obtuvieron los mejores resultados en el rango de movimiento articular de la cadera en comparación con los resultados obtenidos al utilizar el método estático.

Etnyre y Lee (1988) en su estudio concluyen que el método P.N.F., permite mantener por un mayor período la mejora obtenida en el rango de movimiento articular de la cadera; esto es afirmado por Lusting, Ball y
Looney (1992), y por Cornelius, Ebrahim, Watson, Hill (1992), en sus respectivos estudios en los cuales aplicaron solo variaciones en el tiempo en que se ejercía la presión sobre la espalda de los sujetos que utilizaron el método P.N.F. en la articulación de la cadera obteniendo diferencias significativas entre el pre-test y posttest de las diferentes variaciones del P.N.F.; concluyendo también que el método P.N.F. es el más efectivo para mantener por un mayor período la mejora obtenida en el rango de movimiento articular de la cadera.

Debido a la controversia que aún existe sobre la efectividad de los métodos de estiramiento especialmente el método estático y el método P.N.F. al ser aplicados en la articulación de la cadera; surge el interés de seguir investigando sobre éste tema y así extender los resultados ya existentes, los cuales han sido presentados en diferentes estudios; además de estudiar sobre el efecto que tienen ambos métodos en la retención de la mejora obtenida, luego de que se suspende su utilización, lo que aún se ha estudiado muy poco.

El propósito del presente estudio es mostrar el efecto que tienen los métodos de elasticidad estático y P.N.F., en la mejora del rango de movimiento en la articulación de la cadera, y conocer cual de los dos métodos permite mantener por un mayor período de tiempo la mejora obtenida.

\section{METODOLOGÍA}

\section{Sujetos}

Participaron 30 sujetos varones practicantes de nivel principiante del estilo de Kung $\mathrm{Fu}$ Wing Chun cuyas edades estaban comprendidas entre los 15 y 28 años. Los participantes del estudio no conocían, ni tampoco habían realizado anteriormente al estudio alguno de los métodos de estiramiento que se desarrollarían. Se formaron tres grupos de 10 sujetos cada uno, asignados al azar: Grupo A o Control, Grupo B al cual se le aplicaría el método estático y Grupo $\mathrm{C}$ al cual se le aplicaría el método P.N.F. 


\section{Prueba}

Se aplicó la prueba Abdominal Modificada, la cual mide el rango de movimiento articular de la cadera. La prueba Abdominal Modificada se ha utilizado en poblaciones desde los 10 años hasta un nivel universitario. Se ha aplicado de forma satisfactoria en ambos sexos; presentando una confiabilidad de $r=0,92$ cuando fueron correlacionados los puntajes en dos días diferentes y aceptándose una validez por suficiencia.

La Prueba Abdominal Modificada consiste en que los sujetos asuman la posición sentada en el suelo con las piernas extendidas y separadas al ancho de los hombros. En el suelo se coloca una cinta métrica de aproximadamente un metro de longitud, en donde el sujeto se sentará quedando el inicio de la cinta $(1 \mathrm{~cm})$ debajo de la zona de la ingle. Los extremos de la cinta se encontraran debidamente adheridos al suelo con el fin de que la misma no se desplace. Luego de que el sujeto se posiciona sentado en el extremo inicial de la cinta debe tratar de deslizar sobre la cinta métrica una regla, por medio de la flexión de la cadera.

El sujeto debe de realizar lo anterior en tres intentos, y después de cada intento debe de colocarse en la posición inicial. Se toma en cuenta la mayor distancia o medición que realiza.

Con ésta prueba la flexibilidad se mide con la distancia en centímetros que pueda desplazar el sujeto a la regla sobre la cinta métrica.

En las figuras 1,2 y 3 se muestra la posición que deben de asumir los sujetos en el momento de la prueba como en los tratamientos.

\section{Figura 1}

Posición del sujeto en el momento de la prueba desde una perspectiva lateral

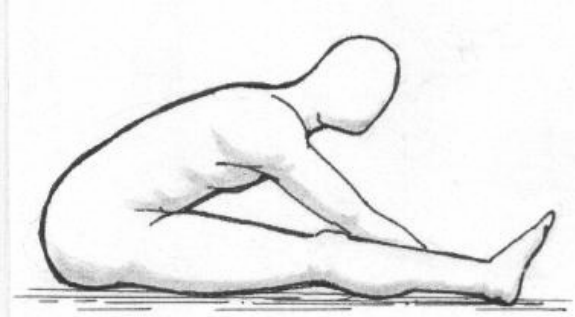

Figura 2

Posición del sujeto en el momento de la prueba desde una perspectiva frontal

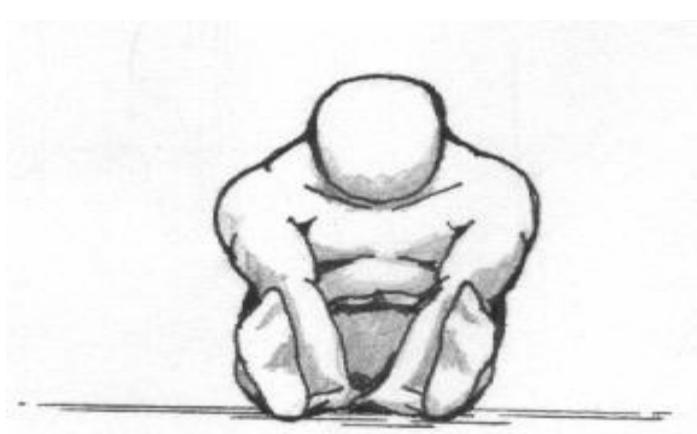

Figura 3

Posición del sujeto en el momento de la prueba desde una perspectiva superior.

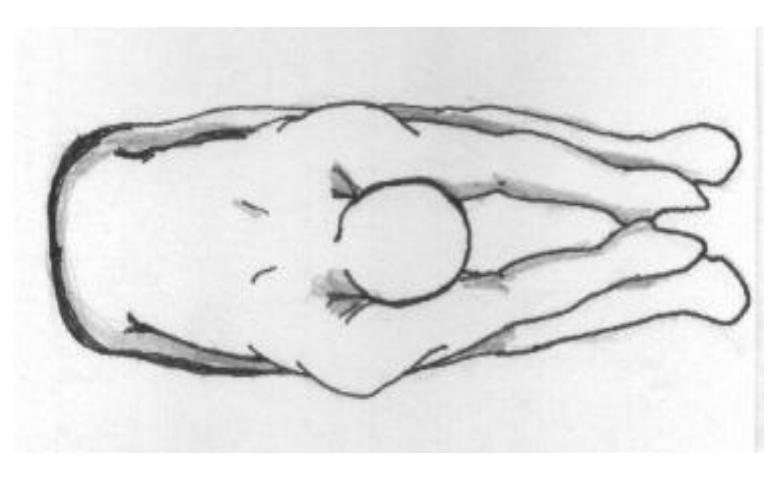




\section{Procedimiento}

El estudio se llevó a cabo durante ocho semanas en las cuales los sujetos del Grupo B y del Grupo $\mathrm{C}$ aplicaron dos veces a la semana, durante los mismos días el tratamiento; el cual lo utilizarían dos veces por sesión. Tales tratamientos eran aplicados al inicio del entrenamiento luego del calentamiento con el objetivo de evitar alguna lesión. El Grupo A prosiguió sus entrenamientos normalmente.

La sesión típica de entrenamiento durante el estudio tuvo una duración de dos horas, en las cuales se realizaba al inicio un calentamiento, utilizando sistemas de circuitos o estaciones; al finalizar el calentamiento se aplicaban los tratamientos de estiramiento específicos a los grupos experimentales. En ésta parte del entrenamiento el Grupo Control no realizaba ningún ejercicio de estiramiento.

Luego de las anteriores actividades se proseguía al entrenamiento técnico-táctico específico de la disciplina marcial.

Antes de iniciar el estudio se les aplicó a cada grupo un pre-test; al finalizar las ocho semanas se aplicó un post-test y al cabo de cuatro semanas se volvió a aplicar un re-test. Los tratamientos se suspendieron durante las últimas cuatro semanas después del post-test; lo anterior para poder verificar mediante el re-test el mantenimiento en la mejora del rango de movimiento de la articulación. Como los dos grupos experimentales se encontraban en el mismo momento en que se aplicarían los tratamientos, los sujetos se formaban en parejas, cuyos integrantes aplicarían el mismo método de estiramiento, además de realizar el mismo calentamiento con el fin de evitar algún tipo de lesión.

Los sujetos que recibían el método estático eran ayudados a flexionar la cadera por un sujeto que utilizaría el mismo tratamiento. Cuando el sujeto que estaba realizando la destreza no podía aumentar el rango de movilidad articular, el ayudante debía ejercer y mantener sobre la espalda una presión constante durante 10 segundos y luego intercambiaban las posiciones. Lo anterior lo realizaban dos veces cada uno.
Los sujetos que recibían el método P.N.F. eran ayudados a flexionar la cadera por un sujeto que utilizaría el mismo tratamiento. En el punto en el cual el sujeto que estaba realizando la destreza no podía aumentar el rango de movilidad articular, el ayudante debía ejercer y mantener una presión constante sobre la espalda durante 10 segundos; luego de finalizar el tiempo, el ejecutante debía de tratar de elevar el tronco durante cinco segundos aún con la presión que le estaba ejerciendo el ayudante. Al finalizar los cinco segundos el sujeto ejecutante finalizaba la elevación del tronco para proseguir con la flexión de la cadera, con la finalidad de que su ayudante volviera a ejercer la presión sobre la espalda durante otros 10 segundos.

Lo anterior lo debían de realizar dos veces para que se tomara como una repetición y poder cambiar de puestos.

La posición adoptada por los sujetos de los grupos experimentales era la misma tanto en el momento en que se aplicaban los diferentes test; como cuando recibían los respectivos tratamientos. Tal posición fue la descrita en la Prueba Abdominal Modificada.

\section{Análisis Estadístico}

Se determinó la Estadística Descriptiva: Promedio (x) y Desviación Estandar (D.S.). También se utilizó un Análisis de Varianza de Dos Vías para Medidas Repetidas, grupos (3 niveles) por mediciones (3 niveles). Un Análisis de Efectos Simples tanto para Grupos como para mediciones y un Análisis de Tukey.

\section{RESULTADOS}

En la Tabla No. 1 se muestran los valores para los promedios y sus respectivas desviaciones estándar (D.S.) obtenidos en el Pre-test, Post-test y Re-test de la prueba de flexibilidad de la cadera, tanto para el grupo control como para los grupos experimentales; donde se puede apreciar los valores más elevados para el Grupo P.N.F. 
Tabla 1

Promedio y D.S. obtenidos en el Pre-test, Post-test y Re-test del Grupo Control como de los Grupos

Experimentales durante la prueba de flexibilidad (cm) de la cadera.

\begin{tabular}{|c|c|c|c|}
\hline \multirow{2}{*}{$\begin{array}{l}\text { Mediciones } \\
(\mathrm{cm})\end{array}$} & Control & Estático & P.N.F. \\
\hline & DS & DS & DS \\
\hline Pre-test & $16,95 \pm 13,08$ & $16,10 \pm 10,41$ & $20,55 \pm 14,85$ \\
\hline Post-test & $17,65 \pm 13,87$ & $23,80 \pm 7,50$ & $29,85 \pm 12,04$ \\
\hline Re-test & $19,55 \pm 13,72$ & $21,70 \pm 8,37$ & $27,55 \pm 15,11$ \\
\hline
\end{tabular}

A los valores obtenidos de los grupos en las diferentes mediciones se les aplicó un Análisis de Varianza de Dos Vías (Anova 3x3, grupos por mediciones), cuyos datos obtenidos se muestran en la Tabla 2.

Tabla 2

Resultados del Anova 3x3.

\begin{tabular}{lcc}
\hline Variables & $\mathrm{F}$ & $\omega^{2}$ \\
\hline Grupos & 1,11 & $0,69 \%$ \\
Mediciones & $35,94 *$ & $4,17 \%$ \\
Interacción & $6,19 *$ & $1,24 \%$ \\
\hline
\end{tabular}

$*: \mathrm{p}<0,05$

El Análisis de Varianza de Dos Vías (Anova 3x3, grupos por mediciones), refleja una significancia para los valores obtenidos de las diferentes mediciones y de la interacción grupos y mediciones; pero no reflejó un valor significativo para los valores obtenidos entre los grupos. Se continuó con un Análisis de Efectos Simples tanto para Grupos como para Mediciones cuyos valores se muestran en las Tablas 3 y 4 respectivamente.

Tabla 3

Resultados del Análisis de Efecto Simple para los Grupos.

\begin{tabular}{|l|c|}
\hline GRUPOS & $\mathrm{F}$ \\
\hline Control & 2,12 \\
Estático & $18,62 *$ \\
P.N.F. & $27,57 *$ \\
\hline \multicolumn{2}{|c|}{$*: \mathrm{p}<0,05$}
\end{tabular}

El Análisis de Efecto Simple aplicado a Grupos reflejó una significancia para los valores obtenidos de los grupos que utilizaron el método Estático y el de Facilitación Propioceptiva
Neuromuscular (P.N.F.) respectivamente; pero no reflejó un valor significativo para el grupo control.

El Análisis de Efecto Simple aplicado a Mediciones reflejó una significancia para los valores obtenidos de las mediciones Post-test y Re-test; pero no reflejó un valor significativo para el Pre-test.

Tabla 4

Resultados del Análisis de Efecto Simple para las Mediciones.

\begin{tabular}{|l|l|}
\hline MEDICIONES & F \\
\hline Pre-test & 6,56 \\
Post-test & $43,72 *$ \\
Re-test & $20,14 *$ \\
\hline
\end{tabular}

$*: \mathrm{p}<0,05$

Luego de realizarse los respectivos análisis de Efecto Simple se prosiguió con los análisis de Tukey tanto para grupos como para mediciones. En el análisis de Tukey realizado a grupos no se encontró una diferencia entre el grupo que utilizó el método estático y el método P.N.F., la diferencia si se mostró en ambos tratamientos con respecto al grupo control. Cuando se aplicó el anterior análisis estadístico a mediciones también se encontró una diferencia en el post-test y re-test al compararse con el pre-test. En la Figura 4 se aprecia el efecto de la interacción de manera que los rangos de mejoría se dan en los grupos que utilizaron tanto el método estático como el método P.N.F., como tratamientos, en comparación al grupo control; el cual se mantiene constante.

En el gráfico se puede apreciar un cambio significativo de pre-test a post-test en los grupos que utilizaron el método de estiramiento estático 
y el método P.N.F. pero no se reflejó un cambio significativo para el grupo control; además de una retención significativa de los resultados obtenidos de los re-test en comparación a los del grupo control.

\section{DISCUSIÓN}

Luego de realizarse los respectivos análisis estadísticos, se pudo verificar la existencia de una diferencia significativa entre los datos obtenidos en el post-test tanto para el grupo que utilizó el método de estiramiento estático como para el grupo que utilizó el método de estiramiento P.N.F. como tratamiento.

Al comparar los datos obtenidos del grupo que utilizó el método de estiramiento estático con respecto al grupo que utilizó el método de estiramiento P.N.F., no se encontró una diferencia significativa entre ambos métodos; pero sí existió una mejoría significativa entre el pre-test y post-test en ambos tratamientos; lo cual también se manifestó en los resultados de los estudios realizados por Lucas y Koslow (1984) y por Williford y Smith (1985).

Los resultados de los diferentes análisis estadísticos también mostraron por medio del pre-test, que los sujetos que utilizaron el método de estiramiento P.N.F. como tratamiento perdieron muy poca de la mejora obtenida en el rango de movimiento de la articulación de la cadera; luego de que se suspendiera el tratamiento durante un mes; lo cual no ocurrió para el grupo de sujetos que utilizó el método de estiramiento estático, en donde la pérdida de la mejora obtenida en el rango de movimiento de la misma articulación fue mayor.

\section{Figura 4}

Efecto de dos diferentes métodos de elasticidad en la mejora del rango de movimiento de la articulación de la cadera

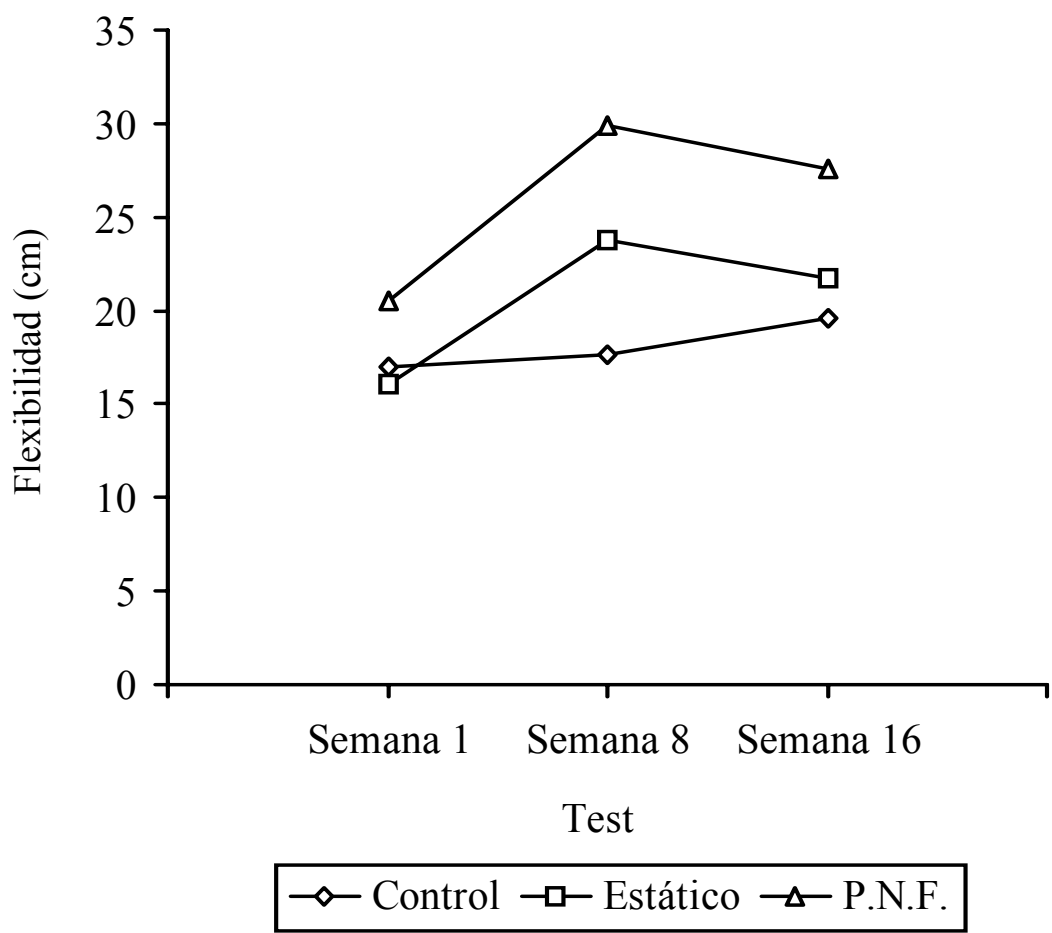

Con lo anterior se confirman los resultados obtenidos en los estudios de Etnyre y Lee (1988), Lusting y colegas (1992); en donde afirman respectivamente que el método P.N.F. permite mantener por un mayor período de tiempo la mejora obtenida en el rango de 
movimiento articular de la cadera, en comparación con el uso del método de estiramiento estático.

Sin lugar a dudas la flexibilidad es un importante componente no sólo del estado físico general; sino también de ser un valioso medio para mejorar el rendimiento durante alguna actividad física o deportiva como por ejemplo en las artes marciales, lo cual es afirmado por Prentice y Bucher (1988). Por lo tanto es importante involucrar en los programas o planes de entrenamiento de diferentes actividades deportivas y especialmente en las artes marciales, métodos de estiramiento como los utilizados en el presente estudio; con la finalidad de mejorar el rango de movimiento de alguna articulación específica.

Se puede concluir que tanto el método de estiramiento estático como el de facilitación propioceptiva neuromuscular (P.N.F.), son igualmente de efectivos para mejorar el rango de movilidad de la articulación de la cadera; además si se desea que la mejora obtenida en la flexibilidad de tal articulación permanezca por más tiempo, se recomienda que el método de estiramiento por utilizar sea el P.N.F.

Por último es importante continuar investigando sobre los efectos que tienen diferentes métodos de estiramiento en la mejora del rango de movimiento en diferentes articulaciones y a su vez en la retención de la mejora obtenida.

\section{BIBLIOGRAFÍA}

Condon, S.; Hutton, R. (1987). Soleus muscle electromyographic activity and ankle dorsiflexion range of motion during four stretching procedures. Physical Therapy. (67) 24-30.
Cornelius, W.; Ebrahim, K.; Watson, J.; Hill, D. (1992). The effects of cold application and modified P.N.F. stretching techniques on hip joint flexibility in college males. Research Quarterly for Exercise and Sport. 63 (3) 311-314.

Etnyre, B.; Lee, E. (1988). Chronic and acute flexibility of men and woman using three different stretching techniques. Research Quarterly for Exercise and Sport. 59 (3) 222-228.

Hardy, L. (1985). Improving active range of hip flexion. Research Quarterly for Exercise and Sport. 56 (2) 111-114.

James, R. y Hart, L. (1990). 100\% en forma. (Primera Edición). Madrid, España: Editorial Augusto E. Pila Teleña.

López, A.(1991). Facilitación neuromuscular propioceptiva. Deporte y Medicina. (12) 9-12.

Lucas R.; Koslow R. (1984). Comparative study of static, dynamic and proprioceptive neuromuscular facilitation stretching techniques on flexibility. Perceptual and motor skills. 58 (2) 615-618.

Lusting, S.; Ball, T. (1992). A comparison of two proprioceptive neuromuscular facilitation techniques for improving range of motion and muscular strength. Isokinetics and exercise science. 2 (4) 154-159.

Magnusson, S.; Simonsen, E.; Aagaard, P.; Boesen, J.; Johannsen, F.; Kjaer, M. (1997). Determinants of musculoskeletal flexibility: viscoelastic properties, cross-sectional area, EMG and stretch tolerance. Scand J. Med. Sci. Sports. (7) 195-202.

Prentice, W.; Bucher, C. (1988). Fitness for college and life. ( $2^{\text {nd }}$ Edic.). E.E.U.U.: Times Mirror. Mosby College Publishing.

Purcell, J.; Hergenroeder, A. (1994). Physical conditioning in adolescents. Curr. Opin. Pediatr. 6 (4) 373-378.

Swinnen, S.; Van Assche, E. (1988). Technieken van lenigheidstraining. Hermes: tijdschrift van het Instituut voor Lichamelijke Opleiding. 19 (2) 167-195.

Williford, H.; Smith, J. (1985). A comparison of proprioceptive neuromuscular facilitation and static stretching techniques. American corrective Therapy journal. 39 (2) 30-33. 